\title{
Adaptive vibration condition monitoring technology for local tooth damage in gearboxes
}

\author{
L. Gelman, \\ Cranfield University, Cranfield, UK \\ 1.gelman@cranfield.ac.uk \\ R. Zimroz \\ Cranfield University, Cranfield, UK \\ r.zimroz@cranfield.ac.uk \\ J. Birkel \\ Turner Powertrain Systems, Wolverhampton,UK \\ Birkel_Jeffrey_F@cat.com \\ H. Leigh-Firbank \\ Caterpillar (UK), Leicester, UK \\ Leigh-Firbank_Howard_C@cat.com \\ D. Simms \\ Peter Brotherhood, Peterborough, UK \\ dsimms@peterbrotherhood.co.uk \\ B. Waterland \\ Peter Brotherhood, Peterborough, UK \\ bwaterland@peterbrotherhood.co.uk \\ G. Whitehurst \\ Turner Powertrain Systems, Wolverhampton,UK \\ Whitehurst_Glyn_D@cat.com
}

Corresponding author: L.Gelman

\begin{abstract}
An adaptive approach was applied for local tooth damage diagnostics in gearboxes. The expediency of adaptation was proved experimentally for the new diagnostic feature, the sum of normalized sideband amplitudes. The positive correlation between mesh amplitudes and their sideband amplitudes was found experimentally for the first time.

Novel adaptive vibration condition monitoring technology for local tooth damage in gearboxes was developed and experimentally validated.

The experimental results showed an increase in effectiveness of the diagnostics when the adaptive technology was used.
\end{abstract}

KEYWORDS: vibration, condition monitoring, gearbox, local tooth damage, adaptation

\section{INTRODUCTION}

Monitoring of gear local tooth damage (e.g. pitting, cracking, spalling, etc.) is extremely important, since a large percentage $(60 \%)$ of gearbox damage is due to gear faults, which in turn are initiated primarily by local tooth damage

Local tooth damage produces a short duration mechanical impact at a comparatively low-energy level. This impact adds modulation effects to the meshing vibration. In turn, these effects produce additional amplitudes of sidebands of mesh harmonics.

Typically, these sidebands are used in performing the local tooth damage diagnostics. Normally, the tooth damage diagnostics is carried out under the presence of the variable nuisance 
parameters of the gearbox, e. g., variable speed and load, vibration interference, etc. To preserve the diagnostics optimality in presence of variable nuisance parameters, the general optimal adaptive approach was proposed [1-3]. The basis of this approach is to measure the variable nuisance parameters and then use adaptive decision-making to decrease the influence of these parameters, thus improving the diagnostic effectiveness. The adaptive decision-making [1-3] depends on the diagnostic feature vector and also on the measurable nuisance parameter vector.

The purposes of this paper are:

- to develop and experimentally validate adaptive vibration condition monitoring technology for local tooth damage in gearboxes based on the above mentioned approach

- to compare experimentally adaptive and non-adaptive technologies

\section{ADAPTIVE CONDITION MONITORING TECHNOLOGY}

The developed technology is based on the amplitude demodulation of the gearbox raw vibrations. We propose to use a new diagnostic feature: the sum of normalized sideband amplitudes. This feature, the accumulated sideband amplitude, is more robust and effective for diagnostics than amplitudes of individual sidebands. We have successfully exploited this accumulation approach for fatigue diagnostics of turbomachinery [4].

The automatic detection of mesh harmonics and sidebands and estimation and normalization of their amplitudes were performed using the automatic "three window" contrast method [3] in frequency domain.

We propose to use simultaneously diagnostic features extracted from fundamental and higher mesh harmonics. This approach also improves diagnostic effectiveness.

Adaptation of the proposed technology can be achieved with the use of the measurable nuisance parameters on which the diagnostic feature depends.

The model of the gearbox mesh harmonic can be written as follows:

$$
x(t)=\left[1+\gamma \sum_{i=1}^{l} s_{i}(t)\right] c(t)+n(t),
$$

where $c(t), n(t),\left\{s_{i}(t)\right\}$ are the steady Gaussian centered uncorrelated mesh harmonic, interference and $l$ narrowband sidebands, $\gamma=\frac{1}{\sqrt{D_{c}}} ; D_{c}$ is the variance of the mesh harmonic.

Taking into account the normality of the signals, $m_{i}=3 \gamma \sigma_{i}$, where $m_{i} \subset[0.1]$ and $\sigma_{i}{ }^{2}$ are the partial modulation coefficient and the variance of the $i-t h$ sideband, respectively.

According to results in ref. [5], the proposed diagnostic feature is proportional to the overall signal/noise ratio of sidebands. Using expression of the autocorrelation function of gearbox vibration $x(t)$, neglecting

- small quantities on the assumption that there is no over modulation

- the cross-effect of the sidebands on the output intereference

the overall signal/noise ratio of sidebands can be written as follows:

$$
Q_{\Sigma}=\frac{q^{2} m_{\Sigma}}{\sqrt{6 \Delta f\left[q^{4}\left(3+2 m_{\Sigma}^{2}\right) \tau_{c}+6 q^{2} \tau_{c n}+3 \tau_{n}\right]}},
$$

where $q$ is the signal/noise ratio of the mesh harmonic; $m_{\Sigma}$ is the overall modulation factor; $\tau_{c}, \tau_{n}$ are quadratic correlation intervals of the mesh harmonic and interference respectively, $\tau_{c n}=\int_{0}^{\infty} R_{c}(\tau) R_{n}(\tau) d \tau, R_{c}(\tau), R_{n}(\tau)$ are the normalized autocorrelation functions of the mesh harmonic and interference respectively, $\Delta f$ is the sideband bandwidth at level $-3 \mathrm{~dB}$. 
This expression is new and generic. It was obtained for mesh harmonic and sidebands with arbitrary autocorrelation functions.

As shown by equation (2), the diagnostic feature depends on the signal/noise ratio with respect to the mesh harmonic, the overall modulation factor, quadratic correlation intervals of the mesh harmonic and interference and sideband bandwidth.

The overall modulation factor is the basic diagnostic parameter. The signal/noise ratio of the mesh harmonic, the quadratic correlation intervals and the sideband bandwidth are the variable nuisance parameters.

The variable nuisance parameters lead to the deterioration of the diagnostic effectiveness [6]. Measurement of

- sideband bandwidth

- quadratic correlation intervals separately for mesh harmonic and interference

are complicated tasks; beyond the scope of this paper.

Here we focus on development of the adaptive technology based on measurable signal/noise ratio of mesh harmonic.

According to results in ref. [5], the normalized mesh amplitude is proportional to the signal/noise ratio of the mesh harmonic.

The development of adaptive technology includes a three stage:

- training stage

- diagnostic stage for each gearbox signal

- diagnostic stage for a set of gearbox signals

Thetraining stage for each gearbox wheel consists of:

- detection of mesh harmonics and estimation of their normalized amplitudes for undamaged and damaged conditions

- estimation of thresholds for normalized mesh amplitudes

- estimation of conditional probability density functions of diagnostic features for damaged and undamaged conditions and for normalized mesh amplitudes located in the selected (by thresholds) ranges

- estimation of adaptive threshold for the diagnostic feature for each range of normalized mesh amplitude

It can be seen that the adaptive threshold for each range of normalized mesh amplitude depends on the conditional probability density functions of the diagnostic feature for damaged and undamaged conditions for this range.

The diagnostic stage for each gearbox vibration signal consists of:

- detection of mesh harmonic and estimation of its normalized amplitude

- comparison of the mesh amplitude with thresholds defined at the training stage

- estimation of the adaptive threshold for the diagnostic feature based on the above comparison

- detection of sidebands and estimation of the diagnostic feature

- decision-making (partial decision) by comparison of the diagnostic feature with the adaptive threshold

The diagnostic stage for a set of gearbox vibration signals consists of the integration of partial decisions using the majority rule.

The majority rule can be described as follows: if the number of partial decisions "faulty" is more than number of partial decisions "undamaged", then the final decision is "faulty" and vice versa.

The schematic of the two diagnostic stages is shown in Fig. 1 for one threshold $T_{\text {mesh }}$ for mesh harmonic and two adaptive thresholds $T_{F s m a l l}$ and $T_{F b i g}$ for the diagnostic feature.

To prove the expediency of the proposed adaptation, we first studied experimentally the correlations between the diagnostic feature and mesh amplitude. 


\section{EXPERIMENTAL SET-UP}

We used two gearboxes for experiments.

The first gearbox is the multistage helical TH-B Powersynchro gearbox (manufacturer is Turner Powertrain Systems (UK)) used in Caterpillar telehandlers. The selected gearbox stage: pinion tooth number is 47 , gear tooth number is 38 , the rotation speed of the pinion is $2000 \mathrm{rpm}$. The gearbox load $500 \mathrm{Nm}$ is provided by output motor. The gearbox vibrations were captured by a $\mathrm{KCF}$ AG107 accelerometer, mounted on the gearbox housing in radial vertical direction. The sensitivity of the accelerometer is $50 \mathrm{pC} / \mathrm{g}$.

The second gearbox is the multistage spur gearbox MkII (manufacturer is Peter Brotherhood, UK) used in Tornado industrial gas turbines. The selected gearbox stage: pinion tooth number is 20 , gear tooth number is 126 , the rotation speed of the pinion is $11085 \mathrm{rpm}$. The gearbox vibrations were captured by the same accelerometer mounted on the gearbox housing in axial direction. The gear load is provided by a two stage blower.

For both gearboxes, we performed the following two sets of experimental trials. Set 1: undamaged gear and pinion of the tested stage; set 2: damaged tooth in gear and pinion of the tested stage. The local tooth damage, pitting, was artificially manufactured by etching the appropriate grooves along the pitch line of a single tooth. Normally, the relative damage size is considered for gearbox monitoring purposes. It is proposed in literature to characterize the relative pitting size by its percentage of the tooth surface area [7]. We manufactured a pitting size of $25 \%$. According to the classification in ref. [7] this pitting size is at level 3, i.e. micropitting and considerable macropitting (15-40\% of the tooth surface); one or more gross pits; damage to both pinion and gear. The pitting is moderately risky on level 3 .

\section{EXPERIMENTAL ESTIMATION OF CORRELATION BETWEEN DIAGNOSTIC FEATURE AND MESH AMPLITUDE}

\subsection{Correlation estimation for helical TH-B Powersynchro gearbox}

The estimation of correlation was performed automatically in the frequency domain, i.e. in the power spectral densities of raw gearbox vibrations. The duration of gearbox signals is $50 \mathrm{~s}$, the frequency resolution is $0.1 \mathrm{~Hz}$, the number of averaged periodograms is 5 .

The main steps of correlation estimation are as follows:

- automatic detection of mesh harmonics and estimation of their normalized amplitudes in power spectral densities of raw vibration signals

- automatic detection of sidebands and estimation of their normalized amplitudes in power spectral densities of raw vibration signals below and above the detected mesh harmonics

- correlation estimation between normalized mesh amplitudes and appropriate sum of normalized sideband amplitudes

The number of signals for damaged gear and pinion is 50. The estimates of normalized correlation coefficients between mesh amplitudes and sum of sideband amplitudes are shown in Fig. 2 for pinion and gear and second and third mesh harmonics.

The number of signals for undamaged gear and pinion is 16 . The estimates of normalized correlation coefficients between second mesh amplitudes and sum of sidebands amplitudes are 0.89 for pinion and 0.95 for gear.

\subsection{Correlation estimation for spur gearbox MkII}

The signal duration is $50 \mathrm{~s}$, the frequency resolution is $0.1 \mathrm{~Hz}$, the number of averaged periodograms is 5 . 
The number of signals for undamaged and damaged gear is 10 and 8 , respectively. The estimates of normalized correlation coefficients between second mesh amplitudes and sum of sidebands amplitudes are $83 \%$ and $70 \%$ for undamaged and damaged gear respectively.

\section{EXPERIMENTAL TRIALS}

The results in paragraph 4 are matched with the obtained expression (2) and show a relatively high level of positive correlation between normalized mesh amplitude and the diagnostic feature for both undamaged and damaged spur and helical gearboxes.

Therefore, the proposed adaptation is expedient, since the diagnostic feature depends on the mesh amplitude.

To estimate the effectiveness of the adaptive technology, we carried out experimental trials using two experimental sets (described above) of TH-B Powersynchro gearbox.

We performed separately (i) the training stage for second mesh harmonic only and damaged and undamaged gear; and (ii) the training and diagnostic stages for several mesh harmonics and damaged and undamaged gear and pinion.

\subsection{The training stage for the second mesh harmonic and damaged and undamaged gears}

The estimated diagnostic features for undamaged and damaged gears for the second mesh harmonic are shown in Fig. 3; the non-adaptive threshold for the diagnostic feature is shown by a continuous black line.

To compare the separation of diagnostic feature at training stage for the adaptive approach vs. non-adaptive approach we have used Fisher's criterion [9]:

$$
F=\frac{\left[m_{1}\left(u / H_{0}\right)-m_{1}\left(u / H_{1}\right)\right]^{2}}{\sigma^{2}\left(u / H_{0}\right)+\sigma^{2}\left(u / H_{1}\right)},
$$

where $u / H_{j}$ is the diagnostic feature for hypothesis $H_{j}$, while $m_{1}$ and $\sigma^{2}$ are the mean and the variance operators respectively.

The value of Fisher's criterion for the non-adaptive case is 4.3.

The normalized amplitudes of the second mesh harmonic for damaged and undamaged gears are shown in Fig. 4 a b respectively. We have used one threshold (value of this threshold is 20) for separation of low and high levels of normalized mesh amplitudes.

The estimated diagnostic features for undamaged and damaged gears and for low and high levels of normalized mesh amplitudes are shown in Fig. 5 a, b. Adaptive thresholds for the diagnostic feature for low and high levels of normalized mesh amplitudes are shown in Fig. 5 by continuous black lines. One can see from Fig. 5 the difference in feature's values and threshold values for low and high levels of normalized mesh amplitudes.

The values of Fisher's criterion are 5.7 and 29.2 for low and high levels of normalized mesh amplitudes respectively. The averaged value of Fisher's criterion for the adaptive case, $F_{a d}=17.5$, is obtained by averaging values of Fisher's criterion for the low and high level of mesh amplitudes.

We consider the gain $R$ in separation of the diagnostic feature at the training stage: the ratio of the averaged Fisher's criterion for the adaptive approach, $F_{a d}$, to the Fisher's criterion $F_{n-a d}$ for the non-adaptive approach:

$$
R=\frac{F_{a d}}{F_{n-a d}}
$$

The obtained estimate of the gain is $R=4.1$. 
Thus, the use of the adaptive approach provides essential gain in separation of the diagnostic feature at the training stage in comparison with the non-adaptive approach. This highlights the expediency of the proposed approach for local damage detection with the new diagnostic feature.

\subsection{The training and diagnostic stages for damaged and undamaged gears and pinions using simultaneously several mesh harmonics}

We have used the second and third mesh harmonics for pinion diagnostics and fundamental, second and third mesh harmonics for gear diagnostics. To test the effectiveness of the competing techniques, the captured gearbox vibration signals were randomly partitioned into 2 sets: one for training purposes $(50 \%)$ and one for diagnostic purposes $(50 \%)$.

For the non-adaptive technology, we performed a training stage for each mesh harmonic and obtained non-adaptive thresholds for diagnostic features. Then we performed the diagnostic stage using the majority rule.

The diagnostic results were successful; the estimates of probability of correct diagnostics for gear and pinion are $100 \%$ :

- undamaged gear diagnostics: 10/0 (i.e. 10 decisions undamaged, 0 decisions "faulty"); faulty gear diagnostics: 0/10 ( 0 decision undamaged, 10 decisions "faulty")

- undamaged pinion diagnostics: 10/0 (i.e. 10 decisions undamaged, 0 decisions "faulty"); faulty pinion diagnostics: 1/9 (1 decision undamaged, 9 decisions "faulty")

For the adaptive technology, we performed the training stage for each mesh harmonic and obtained thresholds for normalized mesh amplitudes and adaptive thresholds for diagnostic features. Then we performed the diagnostic stage using the majority rule.

The diagnostic results are successful; the estimates of probability of correct diagnostics for gear and pinion are $100 \%$ :

- undamaged gear diagnostics: 10/0 (i.e. 10 decisions undamaged, 0 decisions "faulty"); faulty gear diagnostics: 0/10 ( 0 decision undamaged, 10 decisions "faulty")

- undamaged pinion diagnostics: 10/0 (i.e. 10 decisions undamaged, 0 decisions "faulty"); faulty pinion diagnostics: 0/10 (0 decision undamaged, 10 decisions "faulty") We notice that performing diagnostics of faulty pinions using the adaptive technology resulted in no "undamaged" partial decisions, compared to one "undamaged" partial decision using the nonadaptive technology.

These results also show that the proposed adaptive technology provides better effectiveness of local damage detection than the non-adaptive technology.

\section{CONCLUSIONS}

1. The adaptive approach [1-3] was applied to local tooth damage diagnostics in gearboxes. The normalized mesh amplitude was selected as the measurable nuisance parameter.

2. The expediency of the adaptation was proved for the following new diagnostic feature: the sum of normalized sideband amplitudes.

3. The positive correlation between mesh amplitude and diagnostic feature was found experimentally for the first time. This experimental result is matched with the new theoretical result obtained in the paper.

4. To investigate the adaptation effectiveness experimental trials were carried out with two types of multistage gearboxes: spur and helical. The experimental results have shown that an increase of the diagnostics effectiveness was obtained when the adaptive technology was used. These results confirm similar published findings [1-2] and indicate that the use of the proposed adaptive technology improves the local tooth damage diagnostics in gearboxes.

\section{ACKNOWLEDGEMENT}


The financial support of the UK DTI is thankfully acknowledged.

Authors thankfully acknowledge Dr. Combet for covariance calculation (paragraph 4.2)

\section{References}

1. L. M. Gelman Adaptive sequential analysis with variable thresholds. Telecommunications and Radio Engineering 38(9), 75-78, 1983

2. L. M. Gelman, Yu. V. Burau, and V. I. Krytsyn Automatic adaptive method for testing data processing for nondestructive testing in civil engineering. Proceedings of the International Symposium of Nondestructive Testing in Civil Engineering Berlin, Germany 1311-1317, 1995

3. L. M. Gelman, D. A. Kripak, V.V. Fedorov, and L.N. Udovenko Condition monitoring diagnosis methods of helicopter units. Mechanical Systems and Signal Processing 14(4), 613$624, .2000$

4. L. M. Gelman. Bouraou N. I. Passive-active low-frequency vibroacoustical fault diagnosis method, 52 Meeting of the Society for Machinery Failure Prevention Technology, Virginia Beach, USA, 1998

5. L. M. Gelman Estimate of the amplitude modulation factor of noise by a random signal, Telecommunications and Radio Engineering, vol. 47(1), pp. 65-67, 1993

6. V. A. Kovalevsky, Image Pattern Recognition. Springer-Verlag. 1980

7. Fatigue and fracture, ASM Handbook, 1996

8. C. Chen, L. Pau, and P. Wang Handbook on Pattern Recognition and Computer Vision. World Scientific Publishing Company, Inc., 1993

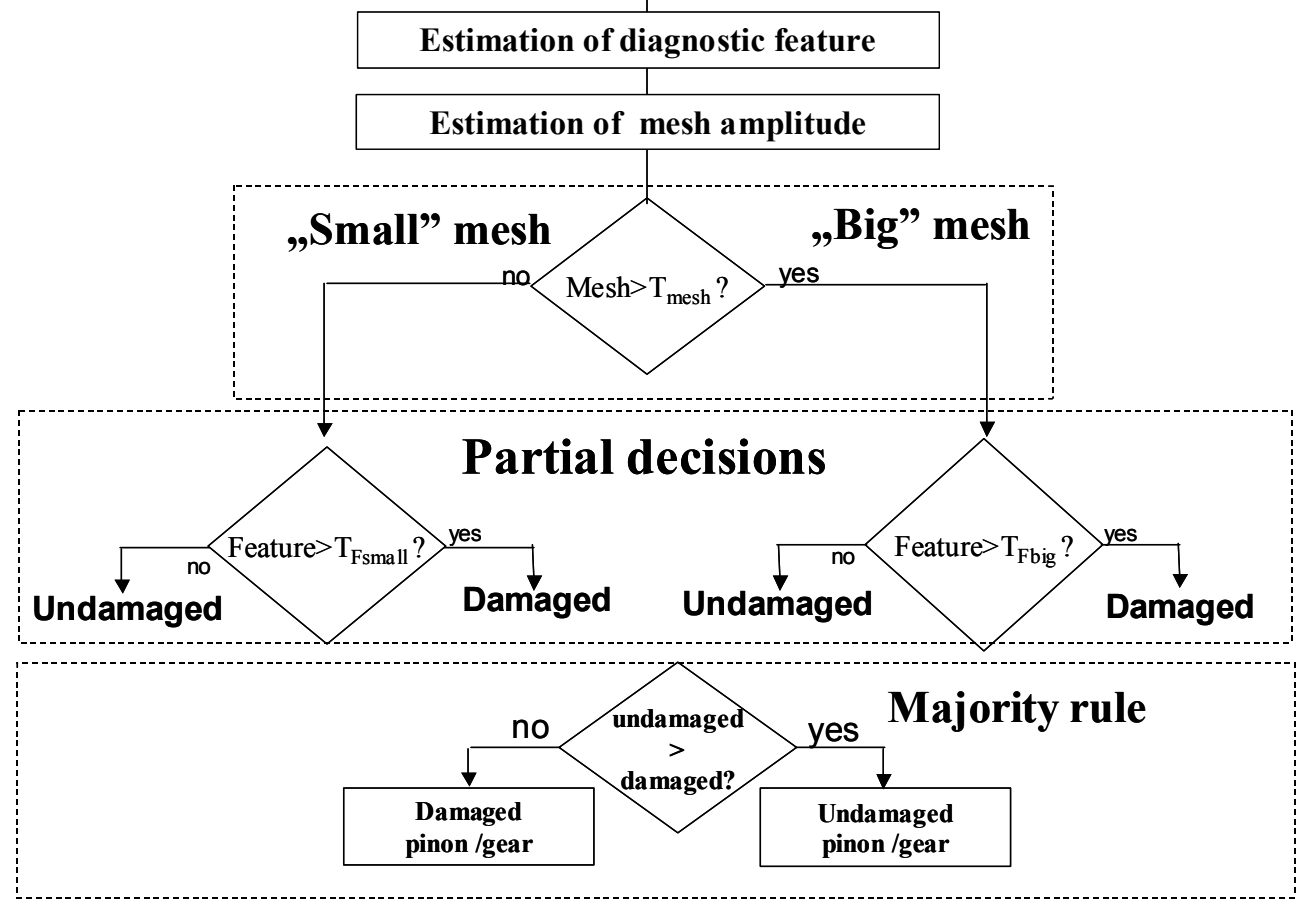

Fig. 1

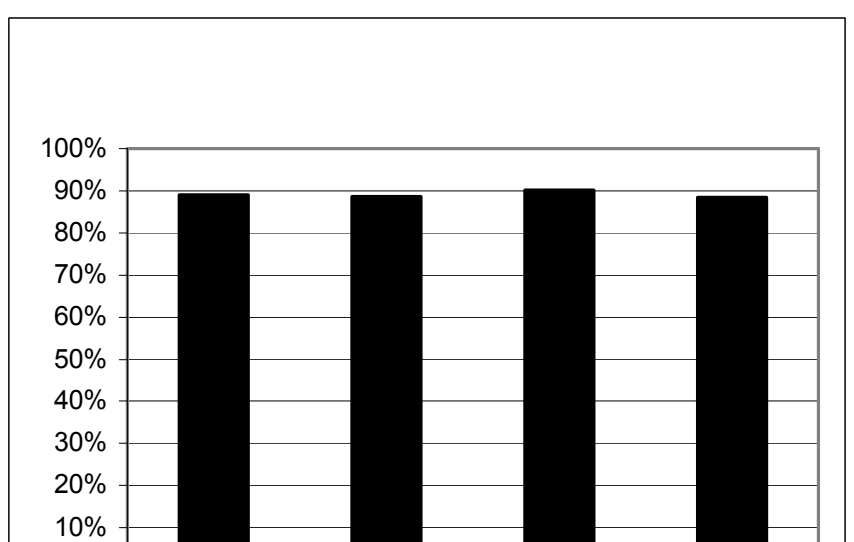


Fig. 2

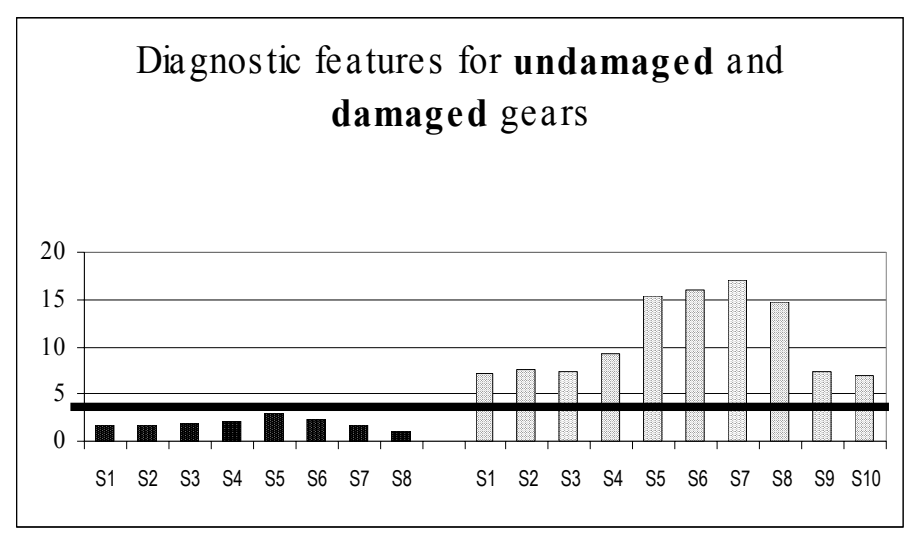

Fig. 3

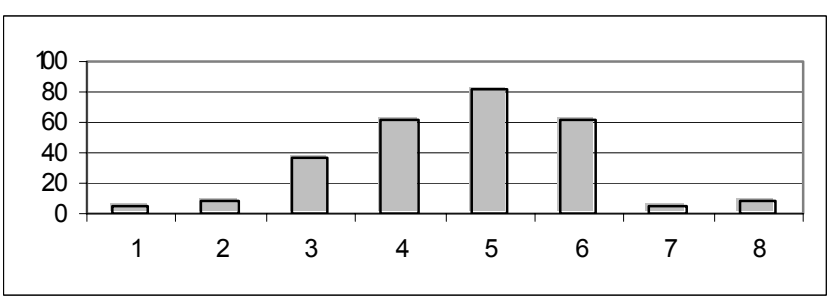

a

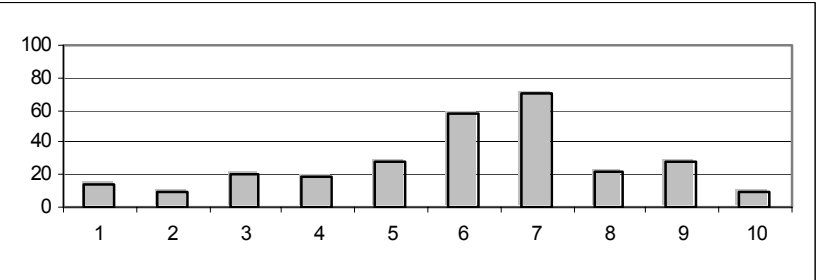

b

Fig. 4

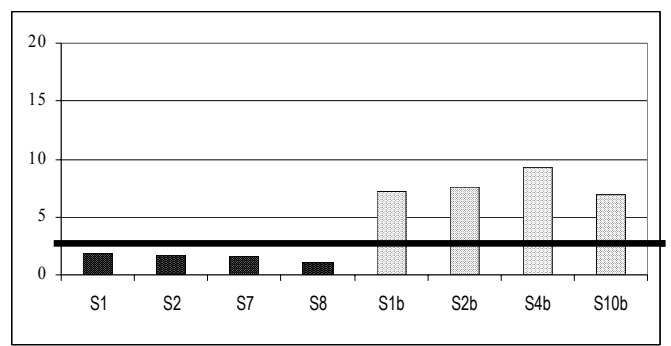

a

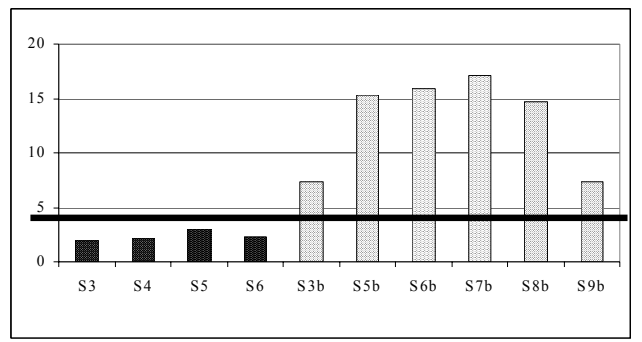

b

Fig. 5 